\title{
circ_TGFBR2 Inhibits Vascular Smooth Muscle Cells Phenotypic Switch and Suppresses Aortic Dissection Progression by Sponging miR-29a
}

\author{
Zhenjun $\mathrm{Xu}^{\mathrm{l}, *}$ \\ Kai Zhong ${ }^{2, *}$ \\ Guanjun Guol,* \\ Can Xu' \\ Zhizhao Song' \\ Dongjin Wang' \\ Jun Pan'
}

'Department of Thoracic and Cardiovascular Surgery, Nanjing Drum Tower Hospital, The Affiliated Hospital of Nanjing University Medical School, Nanjing, 210008, People's Republic of China; ${ }^{2}$ Nanjing Drum Tower Hospital Clinical College of Nanjing Medical University, Nanjing, 210008, People's Republic of China

*These authors contributed equally to this work
Background: Aortic dissection (AD) is a threatening and catastrophic vascular disease with high mortality rate and limited therapeutic strategies. There is emerging evidence showing that circular RNAs play crucial role in regulating various cardiovascular diseases. However, the biological functions and molecular mechanisms of circRNAs in AD still remains elusive. The purpose of this study was to illustrate the potential functional roles and mechanisms of hsa_circ_TGFBR2 in vitro and in vivo.

Methods: The vascular smooth muscle cells (VSMCs) and AD-VSMCs were isolated from normal aorta and AD tissues. The expression of circ_TGFBR2, miR-29a and KLF4 were detected by realtime polymerase chain reaction (RT-PCR) and fluorescence in situ hybridization (FISH). Cell proliferation was assessed by CCK-8 assay, colony formation and EDU assay. Cell migration was evaluated through transwell assay. Dual-luciferase reporter assay and RNA pulldown were performed to identify the interaction between circ_TGFBR2 and miR-29a or between miR-29a and KLF4. The wild-type sequence of circ_TGFBR2 or KLF4 were cloned into the luciferase reporter plasmid, and the activity was measured using dual-luciferase reporter assay system. And for RNA pulldown, the relative RNA enrichment of circ_TGFBR2 and miR29a were confirmed using RT-PCR. Western Blot measured the expression of phenotype switchrelated proteins. $\mathrm{AD}$ rat model induced by $\beta$-aminopropionitrile monofumarate (BAPN) was used to verify the role and mechanism of circ_TGFBR2.

Results: Circ_TGFBR2 inhibited cell proliferation and migration of AD-VSMCs cells. Overexpression of circ_TGFBR2 promoted the expression of contractile markers ( $\alpha$-SMA, SM22 $\alpha$ ) and inhibited the expression of synthetic markers (MGP, OPN) in AD-VSMCs cells. Circ_TGFBR2 served as a sponge for miR-29a targeting KLF4. MiR-29a mimics rescued biological roles induced by circ_TGFBR2 overexpression. The in vivo experiments revealed that overexpression of TGFBR2 suppressed the progression of $\mathrm{AD}$ and increased the expression of contractile markers while inhibited the expression of synthetic markers.

Conclusion: Our study revealed that circ_TGFBR2 regulated VSMCs phenotype switch and suppressed the progression of AD.

Keywords: aortic dissection, circ_TGFBR2, miR-29a, KLF4, vascular smooth muscle cells

\section{Introduction}

Aortic dissection (AD) is an invasive vascular disease with low incidence but high mortality and limited therapeutic treatments. The aortic walls are composed of three layers: the intimal layer, the media and the outer adventitia. ${ }^{1} \mathrm{AD}$ is characterized by a bleeding within the aortic wall or tearing of aortic intimal layer, which leads to separation of the aortic wall layers. ${ }^{2}$ A false lumen is formed during this process 
and it separated from the true lumen by the intimomedial flap. ${ }^{3}$ Quick diagnostic confirmation and appropriate interventional treatment are essential in managing $\mathrm{AD}$ patients. ${ }^{4}$ Vascular smooth muscle cells are crucial in synthesis and contraction of aortic wall in response to various signal stimuli, which mediates vascular remodeling. ${ }^{5}$ During the phenotypic regulation, VSMCs switch from contractile (also termed "differentiated") phenotype to synthetic (also termed "dedifferentiated") phenotype. ${ }^{5,6}$ And synthetic phenotype showed improved viability on cell proliferation, migration, enhanced expression of synthetic markers including OPN (Osteopontin) and MGP (matrix Gla protein), while reduced expression of various differentiation markers such as $\operatorname{SM} 22 \alpha$ and $\alpha$ SMA. Thus, VSMCs phenotype regulation is a significant factor promoting the development of $\mathrm{AD} .{ }^{7,8}$ However, the molecular mechanisms underlying the VSMCs phenotype regulation still remains unknown. Understanding the mechanisms of $\mathrm{AD}$ and find biomarkers may reveal novel diagnostic and therapeutic strategies to prevent $A D$ and reduce clinical mortality.

Circular RNAs (circRNAs) are a novel type of the noncoding RNAs characterized by covalent-closed loop structures that lack of $5^{\prime}$ to $3^{\prime}$ polyadenylated tails, which are formed by backsplicing. ${ }^{9}$ MicroRNAs (miRNAs) are small endogenous noncoding RNAs, which can mediate fundamental cell process through binding to the specific seeds of the 3 'untranslated regions (3'UTRs) of downstream target genes. ${ }^{10}$ CircRNAs were suggested to being related to numerous biological processes and the most common role is acting as miRNAs sponges to regulate target downstream genes expression. ${ }^{11}$ With various researches going on, circRNA was found to be differentially expressed in various types of vascular diseases. Sun et al reported that circACTA2 affected $\alpha$-SMA expression regulated by NRG-1-ICD in VSMCs through NRG-1-ICD/ circACTA2/miR-548f-5p pathway. ${ }^{12}$ CircCBFB acted key roles in VSMCs proliferation and apoptosis at a LYPD3/ GRIA4-dependent way by sponging miR-28-5p in abdominal aortic aneurysm (AAA). GRIA4, LYPD3 and miR-28-5p are essential in circCBRB-mediated VSMCs apoptosis. ${ }^{13}$ Previous researches revealed that knockdown of TGFBR2 gene severely caused acute and sustained aortic pathology including intramural hemorrhage, wall thickening and ulceration. ${ }^{14}$ Prenatal deletion of TGFBR2 inhibited SMCs cells differentiation and interfered TGF- $\beta$ signaling (PMID: 26494233), which caused embryonic death. ${ }^{15}$ However, the biological roles and underlying mechanisms of circRNAs derived from TGFBR2 in AD are largely undisclosed.
In the present research, we identified a down-regulated circular RNA circTGFBR2 in AD tissues and ADVSMCs. Gain and loss of function studies were performed to evaluate the regulatory role of circ_TGFBR2 in VSMCs phenotype switch and AD progression. Mechanistically, we predicted and verified that circ_TGFBR2 could directly sponge miR-29a to suppress Kruppel-Like Factor 4 (KLF4) expression and consequently regulate $\mathrm{AD}$ VSMCs phenotype alteration.

\section{Materials and Methods Specimens and Ethics Statement}

Twenty-five AD aorta samples and normal aorta samples were collected from the Affiliated Drum Tower Hospital of Nanjing University Medical School. The information of donors was shown in Table S1. All experimental procedures were approved by the Ethics Committee of the Affiliated Drum Tower Hospital of Nanjing University Medical School (2020-078-01) and in conformity with the Declaration of Helsinki of the World Medical Association. All tissue donors provided informed consent to have their tissues used in this study. All animal tests were totally agreed with the Guide for the Care and Use of Laboratory Animal by International Committees.

\section{Cell Culture}

The N-VSMCs and AD-VSMCs were obtained from normal aorta tissues and AD tissues as previously described method. ${ }^{16}$ The cells were maintained in DMEM medium (Invitrogen, MA, USA) supplemented with 10\% FBS (Invitrogen, MA, USA) and 1\% antibodies (streptomycinpenicillin, Hyclone). N-VSMCs and AD-VSMCs cells incubation were carried out at $37^{\circ} \mathrm{C}$ in humidified atmosphere containing $5 \% \mathrm{CO}_{2}$. The cells reached a confluent state after 6 to 10 days and then were passaged by $0.05 \%$ trypsin (Invitrogen). The following tests were implemented after 3 to 5 passages.

\section{Cell Transfection}

Specific oligonucleotides and plasmids were designed to regulate the expression of circ_TGFBR2 and miR-29a. Specific siRNAs targeting circ_TGFBR2 were obtained from Sangon Biotech (Shanghai, China). The siRNA sequences were listed in Table S2. The fulllength of circ_TGFBR2 was cloned into pLO5-ciR vector by GenePharma (Shanghai, China) to generated circ_TGFBR2 overexpression plasmids (circ_TGFBR2 
OE) with empty vector as control. The lentiviruses were constructed and packaged by GenePharma (Shanghai, China). The transfection efficiency was shown in Figure $\underline{\mathrm{S} 1}$ (in vitro) and Figure S2 (in vivo). MiR-29a mimics and mimic control were purchased from Sangon Biotech (Shanghai, China) and the transfection efficiency was shown in Figure S3. The sequences were listed in Table

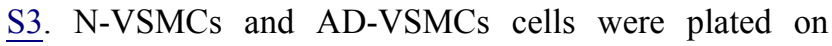
6 -well plates at the density of $8 \times 10^{5}$ and incubated at $37^{\circ} \mathrm{C}$ in DMEM medium free of FBS for 12 hours before transfection. Cell transfection and co-transfection were carried out by Lipofectamine 2000 (Invitrogen, CA, USA) following the manufacturer's protocol. After 6 hours transfection, we replaced the culture medium with DMEM medium containing 10\% FBS. Transfected cells were subjected to RT-PCR analysis after 48 hours.

\section{CCK-8 Assay}

Cell proliferation was assessed by CCK-8 assay (Beyotime, China). Cells were collected and re-suspended in DMEM medium and then plated on 96-well plates at a density of $1 \times$ $10^{4}$ cells/well after treatment. Ten-microliter CCK- 8 regent was added into each well followed by another 2 hours incubation at $37^{\circ} \mathrm{C}$. A microplate Reader (Thermo Fisher Scientific, USA) was used to measure the absorbance at $450 \mathrm{~nm}$. All experiments were tested for 5 times at least.

\section{EDU Incorporation Assay}

We performed EDU assay using Click-it EDU kit (Invitrogen, USA) to analysis DNA synthesis of N-VSMCs and AD-VSMCs. The slides were put into 12well plate and $\times 10^{3}$ cells were seeded in each well. The cells were incubated with $50 \mu \mathrm{mol} / \mathrm{L}$ EDU reagent for 2 hours at $37^{\circ} \mathrm{C}$ and washed with fresh PBS followed by fixation. Then, the cells were stained with $100 \mu \mathrm{L}$ of Apollo reaction cocktail and the nucleus was stained with Hoechst 33342. The fluorescence signals were determined by a fluorescence microscope (Zeiss, Germany). EDU incorporation (\%) =EDU-positive cells/(EDUpositive cells + Hoechst-positive cells) $\times 100 \%$.

\section{Transwell Assay}

Cell migration ability was evaluated through transwell test using $8 \mu \mathrm{m}$ pore filters (Corning, USA). After transfection, $1 \times 10^{5}$ of cells suspended in $200 \mu \mathrm{L}$ DMEM medium were plated on the upper transwell chamber (Corning, USA). Incubated 24 hours at $37^{\circ} \mathrm{C}$, the cells on the surfaces of lower chambers were fixed with $20 \%$ methanol for 10 minutes and dyed with $1 \%$ crystal violet (Beyotime, China). Finally, the migrated cells were counted under the microscope.

\section{Colony-Formation Assay}

After transfection, the cells were seeded in 6 well plates at the density of 700/well. After 14 days of culture, cells were fixed in $10 \%$ formaldehyde and dyed with $1 \%$ crystal violet (Beyotime, China). Images were photographed by a microscope (Leica, Germany) and colonies containing more than 50 cells were counted. All groups were counted at least 5 times.

\section{RealTime Polymerase Chain Reaction}

Total RNA was isolated using TRIzol reagent (Invitrogen, CA, USA) following the manufacturer's protocol. Then, the RNA was reversely transcribed into cDNA using a PrimeScript RT Master Mix Kit (Takara, Dalian, China). Realtime qPCR analysis was conducted using a SYBR-Green real-time PCR mixes (ToyoBo) in a 7900HT PCR System (ABI, CA, USA). All primers used for qRT-PCR were designed and synthesized by Sangon Biotech (Shanghai, China). GAPDH and U6 was used as internal controls for the mRNA/circRNA and miRNA analysis, respectively. Relative expression of genes were calculated by $2^{-\Delta \Delta C t}$ method. All reactions were performed in triplicate. The primers used for realtime PCR were as listed in Table S4.

\section{Western Blot}

The total protein was extracted from VSMCs cells and aorta samples by RIPA lysis buffer (Beyotime, China). The total protein concentration was assessed by BCA protein assay kit (Beyotime, China). Twenty-microgram protein were separated using 10\% SDS-PAGE gels, followed by transfer to polyvinylidene difluoride (PVDF) membranes (Merk, Germany). Subsequently, the blots were blocked in 5\% skimmed milk and then incubated at $4^{\circ} \mathrm{C}$ overnight with primary antibodies including $\alpha$-SMA (1:500, Affinity), SM22 $\alpha$ (1:1000, Affinity), OPN (1:500, Affinity), MGP (1:1000, Proteintech) and GAPDH (1:1000, Affinity) at $4{ }^{\circ} \mathrm{C}$ overnight. After incubation with secondary antibody for 4 hours at room temperature. The bands were visualized using ECL reagents (Pierce, MA, USA) and imaged by the FluorChem imaging system (BioRad Lab, CA, USA). GAPDH was used as the internal control. 


\section{Fluorescence in situ Hybridization (FISH)}

FISH assay was used to ascertain the cellular localization of circ_TGFBR2 and miR-29a. FITC-labeled circ_TGFBR2 probes and Cy3-labeled miR-29a probes were synthesized by GenePharma (Shanghai, China). The sequences of probes were listed in Table S5. FISH experiment was performed using a fluorescent in Situ Hybridization Kit (RiboBio, Guangzhou, China). Briefly, $6 \times 10^{4}$ cells were seeded in 24-well culture medium and incubated for 24 hours. Then, cells fixed with $4 \%$ paraformaldehyde at room temperature for 20 minutes and washed with $0.5 \%$ Triton X-100. Besides, $4 \mu \mathrm{mol} / \mathrm{L}$ probes for circ_TGFBR2 and miR-29a were added into ADVSMCs cells and followed by incubation in the dark overnight. At last, cell nuclei were stained with DAPI and captured using confocal laser-scanning microscopy (Zeiss, Germany).

\section{RNA Pull-Down Assay}

Biotin-labeled miR-29a or KLF4 probe and the NC probe were synthesized by RiboBio (Guangzhou, China). $1 \times 10^{6}$ AD-VSMCs cells were harvested and lysed. Probe-coated beads were generated by incubation with miR-29a or KLF4 probe and C-1 magnetic beads (Life Technologies, USA). Cells lysate with miR-29a or KLF4 probe or NC probe were cultured for 24 hours $4{ }^{\circ} \mathrm{C}$. At last, the pull down complex was eluted and subjected to RT-qPCR analysis.

\section{Immunohistochemistry (IHC)}

The aorta tissues were fixed and embedded in paraffin followed by cutting into $4 \mu \mathrm{m}$ sections. Tissue sections were deparaffinized in xylene, rehydrated in graded ethanol. Slides were blocked by $2.5 \%$ horse serum for 1 hour at room temperature and followed incubated with primary antibodies (Ki67, 1:500, Affinity) at $4^{\circ} \mathrm{C}$ overnight. After incubation with HRP-conjugated goat anti-mouse secondary antibody at $37^{\circ} \mathrm{C}$ for 20 minutes, the sections were measured using a light microscope (Leica, Germany) and pictured.

\section{Dual-Luciferase Reporter Assay}

The wild-type (WT) circ_TGFBR2, Mutant-type (MUT) circ_TGFBR2, WT KLF4 and MUT KLF4 plasmids were constructed by Sangon Biotech (Shanghai, China). The plasmids and miR-29a mimics or NC control were cotransfected into AD-VSMCs cells. After $48 \mathrm{~h}$ transfection, cells were harvested and analyzed through a DualLuciferase Reporter Assay kit (Promega, CA, USA). The relative luciferase activity was measured by a GloMax fluorescence reader (Promega, CA, USA). Renilla luciferase activity was used to normalize the differences in transfection efficiency. Each assay was carried out in 5 times at least.

\section{Aortic Dissection Rat Model}

$\beta$-Aminopropionitrile monofumarate (BAPN) is widely reported to induce $\mathrm{AD}$ disease in animals through restraining the cross-links in collagen and elastin fibers. ${ }^{17,18}$ We conducted the AD model as previously described. ${ }^{19,20}$ SpragueDawley rats (3 weeks old) were fed with a normal diet and administrated orally $0.25 \%$ BAPN (Sigma, St, Louis, USA) to 7 weeks old for the AD model group. And the Sham group was treated the same volume of saline. Then, at 6 weeks old, the $\mathrm{AD}$ model rats were divided into the following five groups at random: Model group $(\mathrm{n}=5)$, Len-vector group $(\mathrm{n}=5)$, Len-si-NC group $(\mathrm{n}=5)$, Len-circ_TGFBR2 overexpression group $(n=5)$ and Len-si-circ_TGFBR2 group $(n=5)$. The circ_TGFBR2 overexpression group, the vector group, the si-NC group and the si-circ_TGFBR2 group were respectively injected with lentivirus carrying circ_TGFBR2 OE, control vector, si-NC and si-circ_TGFBR2 $\left(1 \times 10^{11}\right.$ PFU/rat) via tail vein. Rats that died during the period were autopsied at that moment immediately, while the living rats were killed via an overdose of anesthetic sodium pentobarbital intravenously at the endpoints of the experiments. The aorta tissues were collected and immediately fixed into 4\% paraformaldehyde (PFA) solution for following assay. Animal experiments were performed according to the Guide for the Care and Use of Laboratory Animals.

\section{Statistics Analysis}

All statistical analyses were interpreted by GraphPad prism 7.0 version (GraphPad Software, CA, USA). The results are represented as mean \pm standard deviation (SD). Statistical significance was set at $\mathrm{P}<0.05$ detected by 2-tailed independent Student's $t$-test for comparisons between two groups, 1-way or 2-way AVONA, followed by Tukey's post-hoc test for multiple comparisons. Pearson analyses were carried out to assess the correlation.

\section{Results \\ circ_TGFBR2 Was Relatively Down-Regulated in AD}

By examining the UCSC database, we discovered that the 1302-bp circ_TGFBR2 was generated by circularization of exon 4 of TGFBR2 gene with the position of chr3:30686238-30715738. cDNA and genomic DNA were extracted from AD-VSMCs cells and then were subjected 
to gel electrophoresis. The results revealed that circ_TGFBR2 could be detected in cDNA while no amplification product was observed in gDNA (Figure 1A). Whereafter, the stability of circ_TGFBR2 was determined in AD-VMSCs cells by RNase R. The results showed that RNase $\mathrm{R}$ degraded the linear transcript of TGFBR2, while the circular transcript of circ_TGFBR2 was resistant to digestion with RNase R, implying that circ_TGFBR2 was circular (Figure 1B). Besides, we evaluated the expression level of linear TGFBR2 and circ_TGFBR2 with the treatment of actinomycin D. The results showed that the level of circ_TGFBR2 had no significant change with actinomycin $\mathrm{D}$ treatment at different times. While the linear TGFBR2 expression was largely decreased at $8 \mathrm{~h}$, 12h, 24h after actinomycin D treatment (Figure S4). In addition, FISH experiment revealed that circ_TGFBR2 was down-regulated in in clinical AD aorta samples compared with normal aorta tissues (Figure 1C and D). Moreover, Figure 1E showed that circ_TGFBR2 located mainly in cytoplasm in AD-VSMCs cells. Additionally, the qRT-PCR data evaluated that circ_TGFBR2 was remarkably down-regulated in clinical AD samples compared to the normal aorta tissues (Figure 1F).

\section{Inhibition of circ_TGFBR2 Promoted the Proliferation, Migration and Phenotype Switch in N-VSMCs Cells}

Firstly, we determined the expression of circ_TGFBR2 between N-VSMCs cells and AD-VSMCs cells. The RTPCR results showed that circ_TGFBR2 was significantly down-regulated in AD-VSMCs cells, that was consistent with the conclusions tested in clinical samples (Figure 2A). Specific siRNA targeting circ_TGFBR2 was synthesized and the interference efficiency was
A
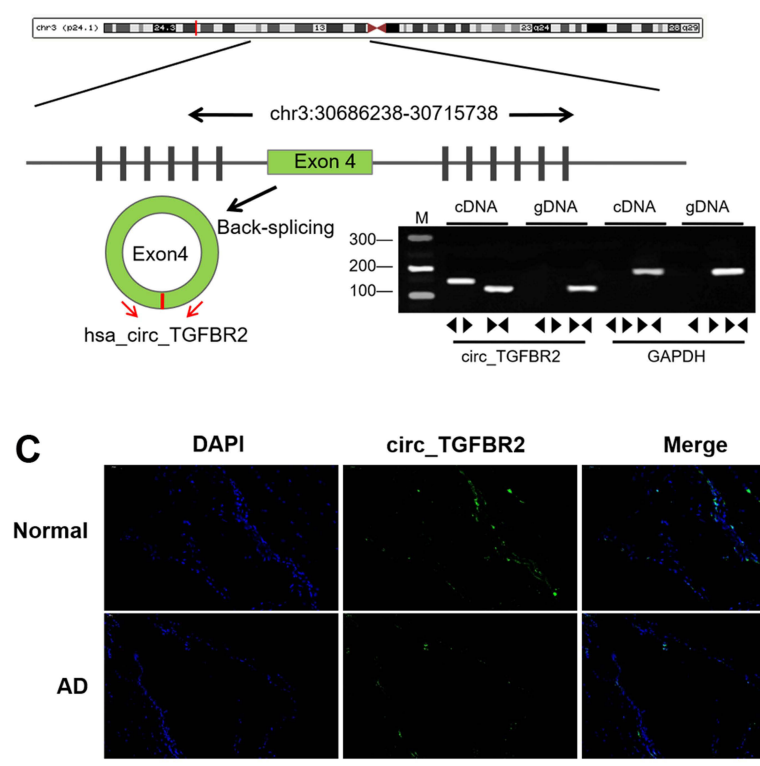

E

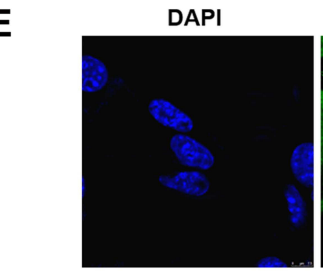

circ_TGFBR2

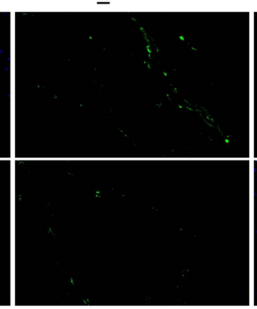

circ_TGFBR2

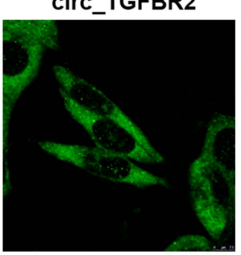

Merge
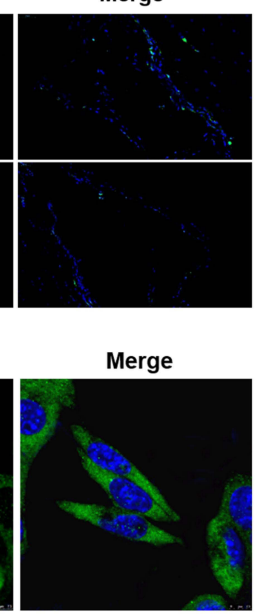

B

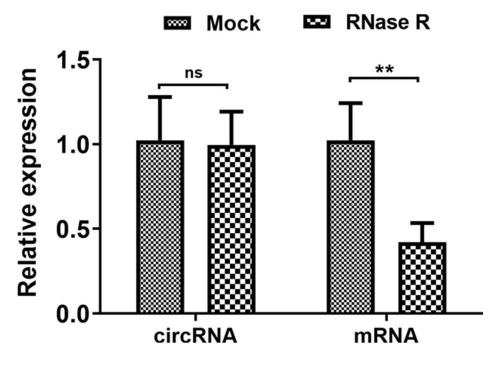

D

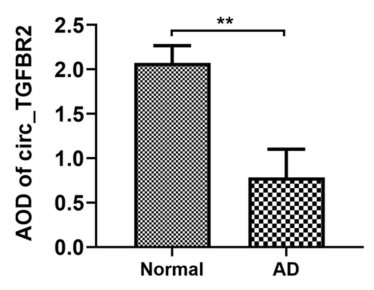

$\mathbf{F}$

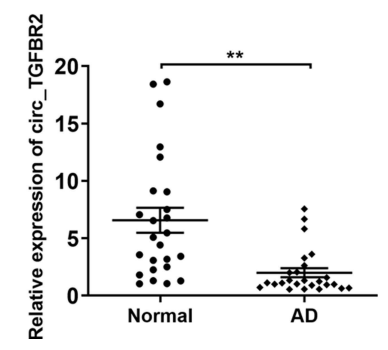

Figure I Biological characterization of circ_TGFBR2 in Aortic dissection (AD). (A) circ_TGFBR2 was generated by the cyclization of exon 4. Gel electrophoresis was applied to show the expression level of circ_TGFBR2 and GAPDH transcript. circ_TGFBR2 was amplified by divergent primers in cDNA rather than genomic DNA. (B) QRT-PCR analysis verified that linear TGFBR2 could be digested by RNase R, whereas circ_TGFBR2 was resisted to RNase R treatment ( $n=3)$. (C and $\mathbf{D})$ Situ hybridization experiment was performed to determine the expression of circ_TGFBR2 in clinical normal aorta and AD tissues $(n=3)$. Scale bar $=50 \mu$ m. $(\mathbf{E})$ Situ hybridization experiment was carried out to explore the location of circ_TGFBR2 in AD-VSMCs cells $(n=3)$. Scale bar $=20 \mu \mathrm{m}$. (F) The expression level of circ_TGFBR2 in AD tissues and normal aorta samples $(\mathrm{n}=25)$. **p $<0.01$, $\mathrm{ns}=$ non-significant in 2-tailed Student's $t$-test $(\mathbf{B}, \mathbf{D}$ and $\mathbf{F})$.

Abbreviation: $A D$, aortic dissection. 
A

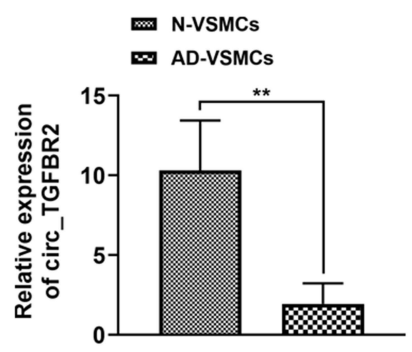

D

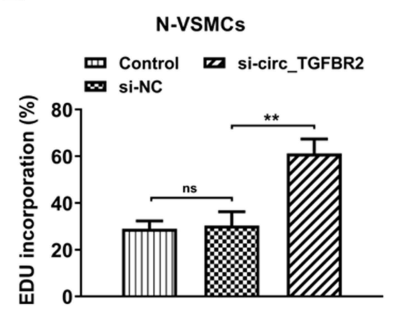

G
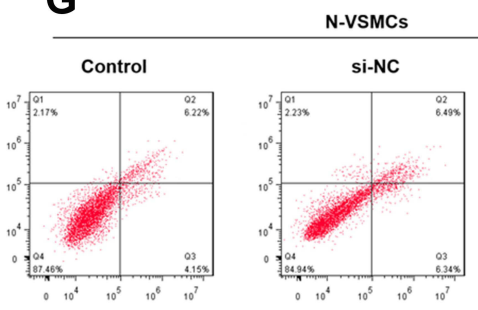

J

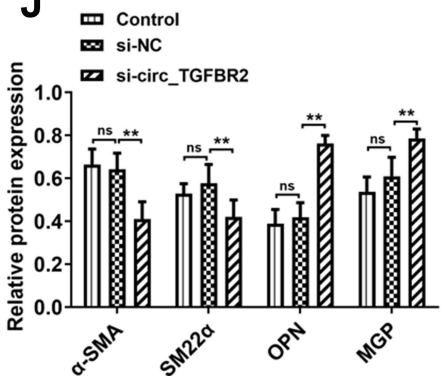

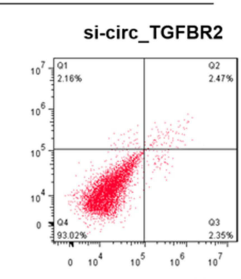

B

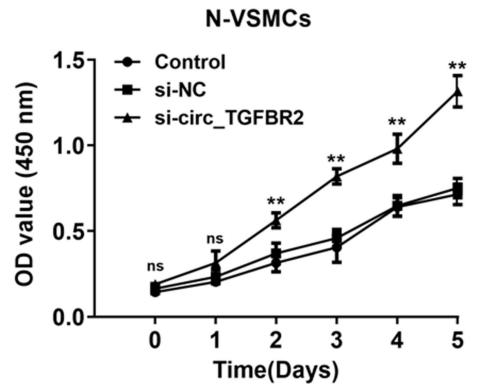

E
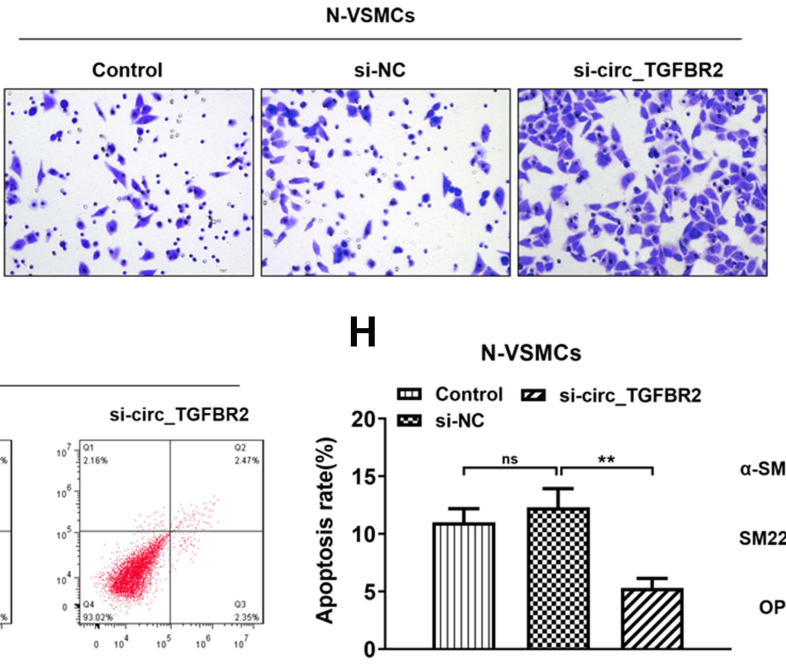

C EDU

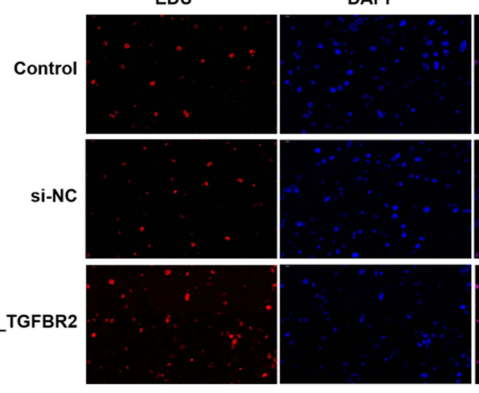

F

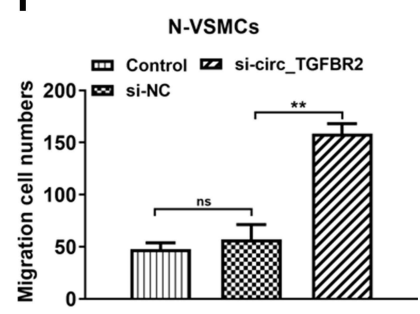

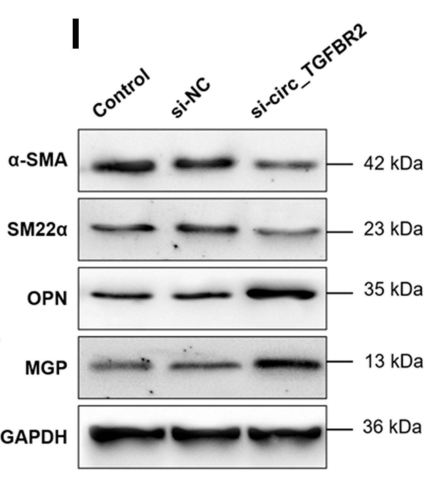

Figure 2 Inhibition of circ_TGFBR2 enhanced the proliferation, migration and phenotype switch of N-VSMCs cells. (A) The expression of circ_TGFBR2 was downregulated in AD-VSMCs cells compared with N-VSMCs cells $(n=3)$ through qRT-PCR analysis. (B-D) CCK-8 and EDU staining were carried out to explore the cell proliferation of N-VSMCs cells after si-circ_TGFBR2 transfection $(n=3)$. Scale bar $=20 \mu \mathrm{m}(\mathbf{E}$ and $\mathbf{F})$ Transwell assay was applied to determine the migration ability of $\mathrm{N}-$ VSMCs cells that inhibited the expression of circ_TGFBR2 $(n=3)$. Scale bar $=20 \mu \mathrm{m}$. $(\mathbf{G}$ and $\mathbf{H})$ Flow cytometry was performed to measure cell apoptosis $(n=3)$. $(\mathbf{I}$ and J) Phenotype switch-related proteins expression of N-VSMCs cells was determined via Western Blot analysis $(n=3)$. The levels of $\alpha$-SMA, SM22 $\alpha$, OPN and MGP were normalized to GAPDH levels. ${ }^{* *} p<0.01$, ns=non-significant in 2-tailed $t$-test $(\mathbf{A})$ and Tukey's post-hoc comparisons test (B, D, F, $\mathbf{H}$ and $\left.\mathbf{J}\right)$.

Abbreviations: N-VSMCs, vascular smooth muscle cells from normal aorta; AD-VSMCs, vascular smooth muscle cells from AD aorta; $\alpha$-SMA, alpha-smooth muscle actin; SM22 $\alpha$, smooth muscle protein 22 $\alpha$; OPN, Osteopontin; MGP, Matrix Gla protein; GAPDH, Glyceraldehyde-3-phosphate dehydrogenase; EDU, 5-ethynyl-2'-deoxyuridine.

determined by RT-PCR (Figure S5). si-circ_TGFBR2-2 was selected for further research. We performed CCK-8 and EDU assay to evaluate the proliferation in $\mathrm{N}-\mathrm{VSMCs}$ cells. The results indicated that inhibition of circ_TGFBR2 remarkably enhanced cell proliferation (Figure 2B-D). Moreover, transwell assay and flow cytometry analysis demonstrated that N-VSMCs cells migration ability was accelerated while the apoptosis was significantly reduced after si-circ_TGFBR2 transfection (Figure 2E-H). Besides, Western blot results revealed that inhibition of circ_TGFBR2 remarkably decreased the expression of contractile markers $(\alpha-$ SMA, SM22 $\alpha$ ) and increased the expression of synthetic markers (MGP, OPN) (Figure 2I and J), which demonstrated that inhibition of circ_TRGFBR2 promoted the phenotype switch in N-VSMCs cells. 


\section{Overexpression of circ_TGFBR2}

Inhibited the Proliferation, Migration, and Phenotype Switch of AD-VSMCs Cells

To investigate the biological functions of circ_TGFBR2 in AD-VSMCs cells, we cloned the circ_TGFBR2 sequence into pLO5-ciR vector to generate circ_TGFBR2 overexpression plasmids. Firstly, qRT-PCR assay was carried out to evaluate the expression of circ_TGFBR2 and linear TGFBR2 after transfection with vector or circ_TGFBR2 overexpression plasmids. The results indicated that the expression of circ_TGFBR2 was obviously enhanced in the circ_TGFBR2 OE group compared to the vector group whereas the level of linear TGFBR had no significant change in AD-VSMCs cells (Figure S6). CCK-8 assay and EDU assay were carried out to explore the proliferation of AD-VSMCs cells. A notable inhibition of cell proliferation after circ_TGFBR2 overexpression plasmids transfection was observed (Figure $3 \mathrm{~A}-\mathrm{C}$ ). Additionally, circ_TGFBR2 overexpression remarkably reduced cell migration ability and enhanced cell apoptosis (Figure 3D-G). Moreover, Western blot analysis was carried out to examine phenotype switch-related proteins expression of AD-VSMCs cells after plasmid transfection. The results showed that overexpression of circ_TGFBR2 obviously promoted the expression of contractile markers ( $\alpha$-SMA, SM22 $\alpha$ ) and inhibited the expression of synthetic markers (MGP, OPN) in AD-VSMCs cells (Figure 3H and I).

\section{circ_TGFBR2 Functioned as a miR-29a Sponge}

Previous studies have determined that circRNAs sponge miRNAs to participate in the regulation of cell
A

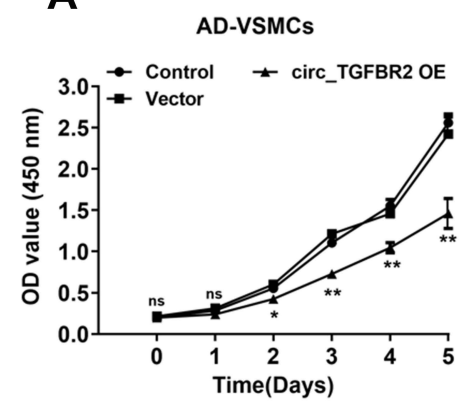

D

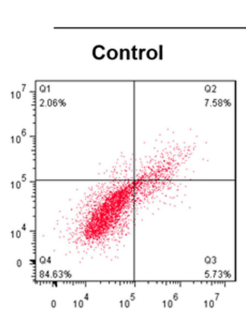

AD-VSMCs

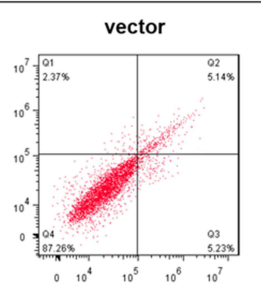

G

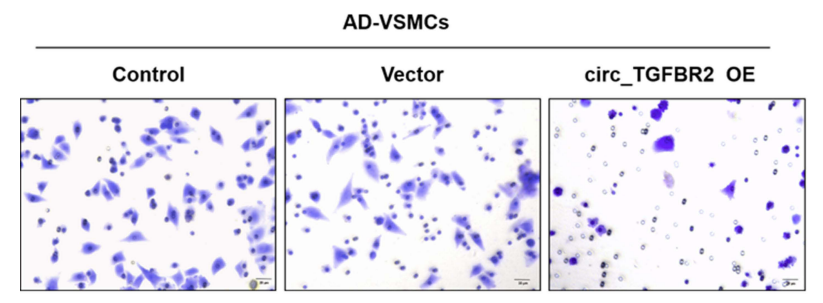

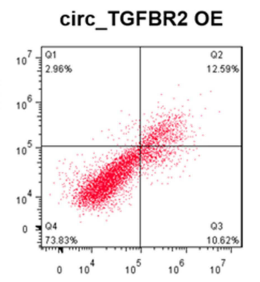

B
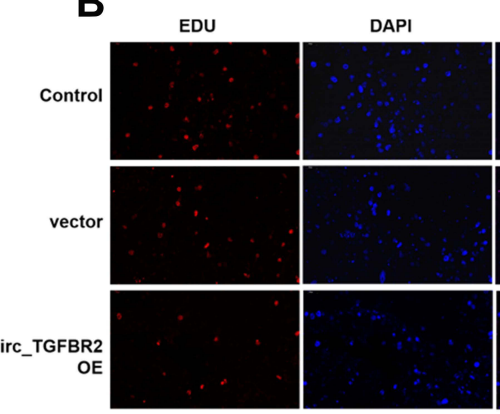

E
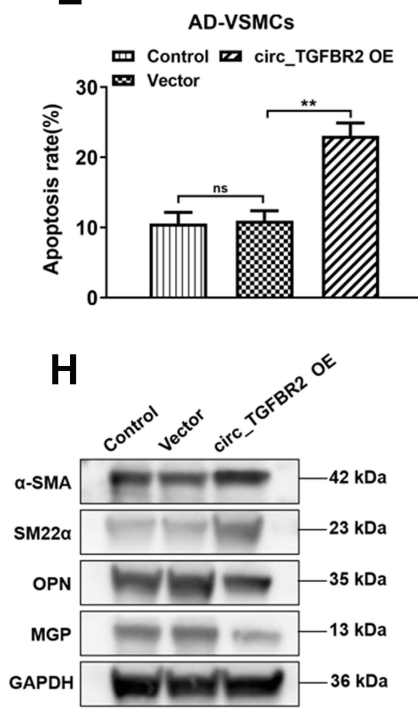

C

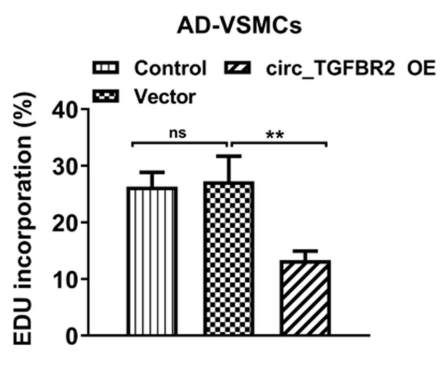

$\mathbf{F}$

AD-VSMCs

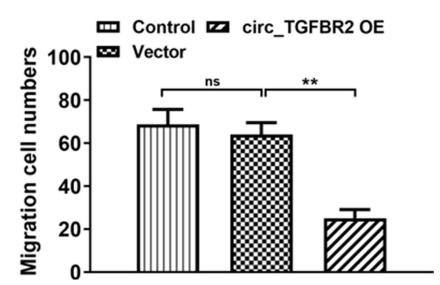

I AD-vsmCs

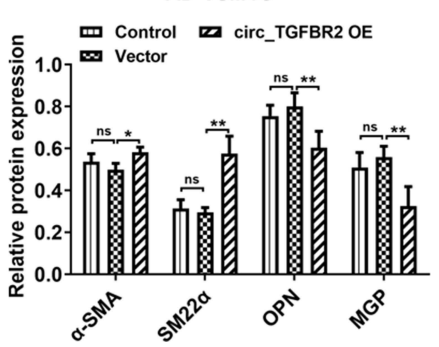

Figure 3 Overexpression of circ_TGFBR2 regulated the proliferation, migration, apoptosis and phenotype switch of AD-VSMCs cells. (A-C) The cell proliferation of ADVSMCs cells was reduced after circ_TGFBR2 overexpression plasmids transfection determined by CCK-8 and EDU staining ( $\mathrm{n}=3$ ). Scale bar $=20 \mu \mathrm{m}$. (D and $\mathbf{E})$ The cells apoptosis was increased that overexpressed circ_TGFBR2 compared with the vector group via Flow cytometry $(n=3)$. (F and $\mathbf{G})$ Transwell assay was applied to determine the migration ability of AD-VSMCs cells treated as indicated $(n=3)$. Scale bar $=20 \mu \mathrm{m}(\mathbf{H}$ and $\mathbf{I})$ Phenotype switch-related proteins expression of $A D-V S M C s$ cells was determined using Western Blot analysis $(\mathrm{n}=3)$. ${ }^{*} p<0.05,{ }^{*} p<0.0 \mathrm{I}$, ns $=$ non-significant in Tukey's post-hoc comparisons test $(\mathbf{A}, \mathbf{C}, \mathbf{E}, \mathbf{F}$ and $\mathbf{I})$.

Abbreviation: AD-VSMCs, vascular smooth muscle cells from AD aorta; $\alpha$-SMA, alpha-smooth muscle actin; SM22 $\alpha$, smooth muscle protein $22 \alpha$; OPN, Osteopontin; MGP, Matrix Gla protein; GAPDH, Glyceraldehyde-3-phosphate dehydrogenase; circ_TGFBR2 OE, circ_TGFBR2 overexpression plasmids. 
physiology process. ${ }^{21}$ Therefore, bioinformatics analysis (circular RNA interactome) was screened to predict the miRNAs which potentially target circ_TGFBR2 (Figure S7). Cells were transfected with different kind of miRNA mimics. The luciferase activity assay indicated that miR29a showed the highest luciferase suppression efficiency (Figure 4A). Otherwise, bioinformatics analysis showed the miR-29a binding site with circ_TGFBR2 (Figure 4B). Then, Luciferase reporter assay was carried out. The findings indicated that miR-29a only notably decreased the relative luciferase intensity of circ_TGFBR2-WT group compared with other groups (Figure 4C). To further confirm the interaction of circ_TGFBR2 and miR-29a, RNA pull down was performed in ADVSMCs cells. As is shown in Figure 4D, biotin-labeled miR-29a enriched circ_TGFBR2, thus indicating the binding interaction of circ_TGFBR2 and miR-29a. Additionally, FISH assay revealed that circ-TGFBR2 was co-localized in cytoplasm with miR-29a in ADVSMCs cells (Figure 4E). The qRT-PCR results showed that miR-29a was obviously up-regulated in clinical AD samples compared to the normal aorta samples (Figure 4F). Besides, the Pearson analysis revealed the negative correlation between circ_TGFBR2 and miR-29a (Figure 4G).

\section{miR-29a Effectively Reversed the}

\section{Function of circ_TGFBR2 in AD}

We next explored whether circ_TGFBR2 regulated AD progression through miR-29a. As shown in Figure 5A, compared to the control vector group, circ_TGFBR2 overexpression plasmids group inhibited proliferation of ADVSMCs cells, yet the miR-29a mimics group increased proliferation. Co-transfection of circ_TGFBR2 overexpression plasmids and miR-29a mimics eliminated the influence on cell proliferation caused by only circ_TGFBR2 overexpression plasmids. Furthermore, function studies revealed that circ_TGFBR2 overexpression could remarkably inhibit the clonality, migration and raised apoptosis of AD-VSMCs cells. On the contrary, miR-29a mimics transfection notably improved the clonality, migration and decreased apoptosis od AD-VSMCs cells. Co-transfection of circ_TGFBR2 overexpression plasmid and miR-29a mimics reversed the influence of upregulated circ_TGFBR2 on clonality, migration and apoptosis of AD-VSMCs cells. (Figure 5B-G). In addition, Western blot results indicated that co-transfection of circ_TGFBR2 overexpression plasmid and miR-29a mimics overturned the impact of up-regulated circ_TGFBR2 on the expression of contractile markers $(\alpha-$

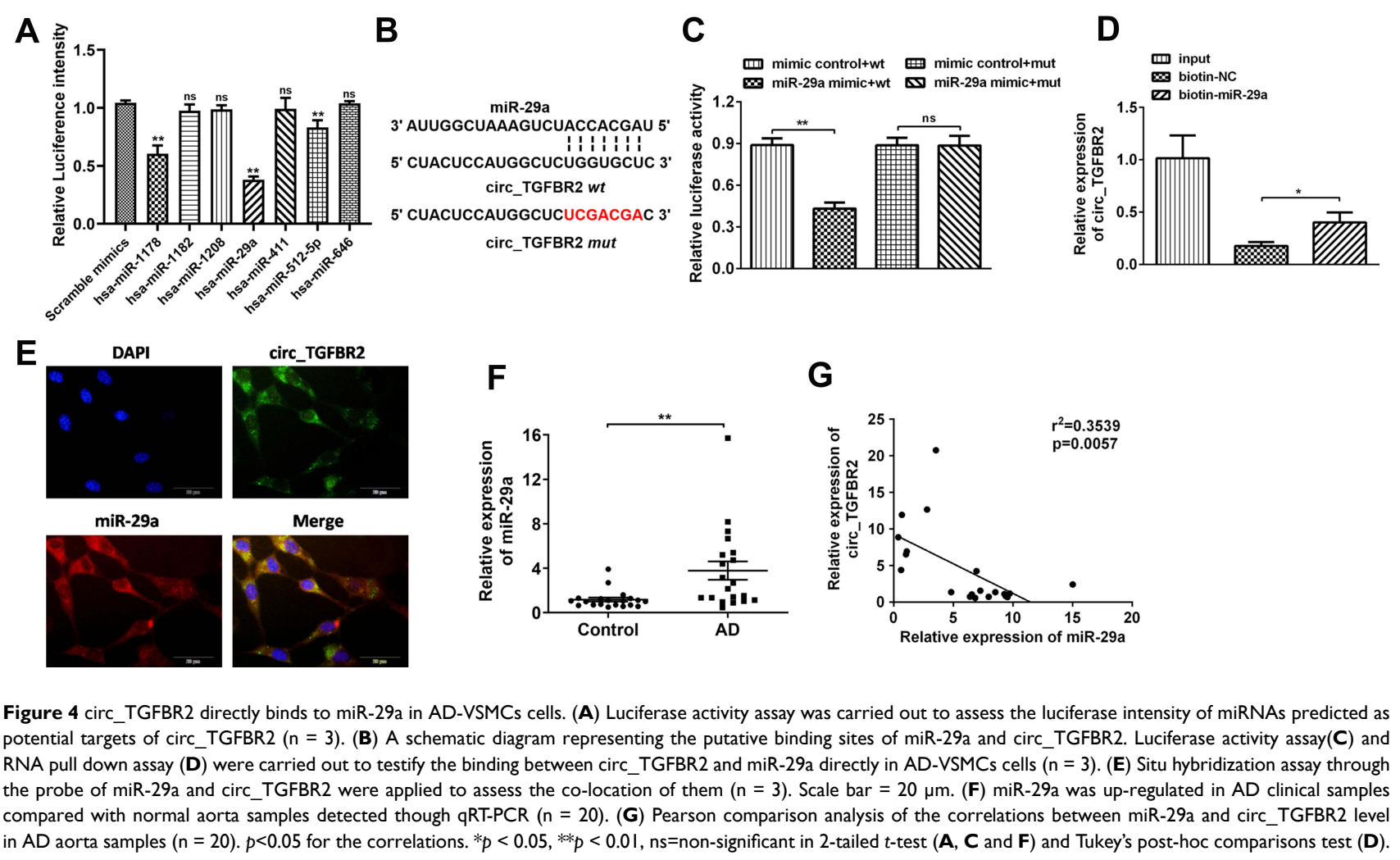


A

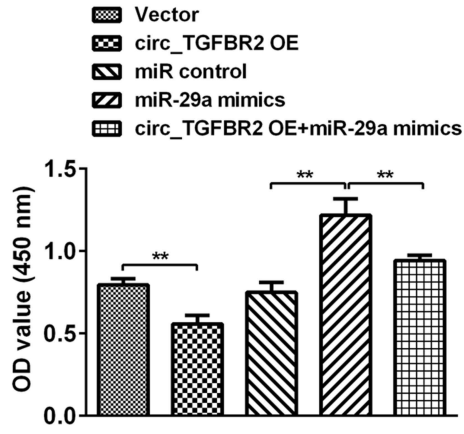

B

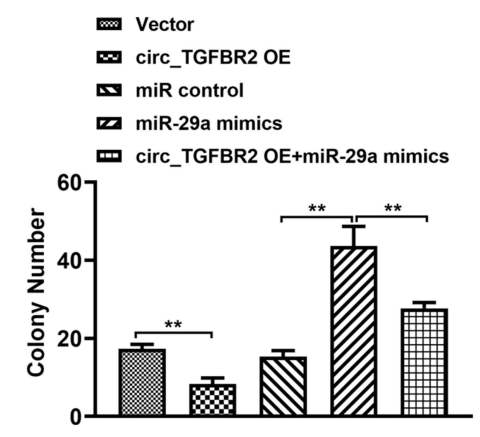

E

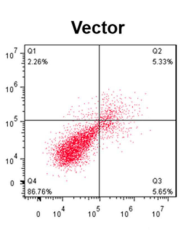

miR-29a mimics

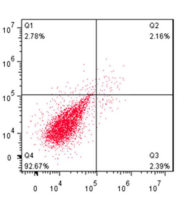

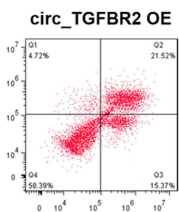
circ_TGFBR2 OE+
miR-29a mimics

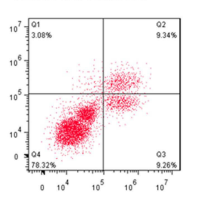

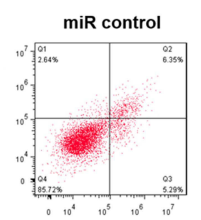
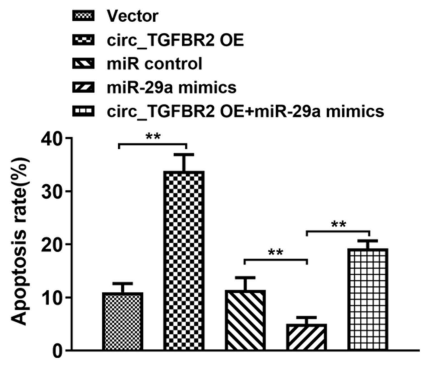

C

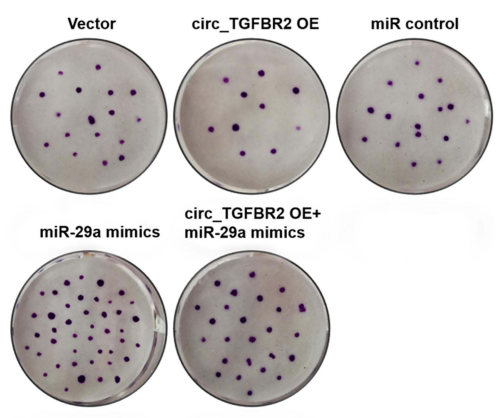

$\mathbf{F}$
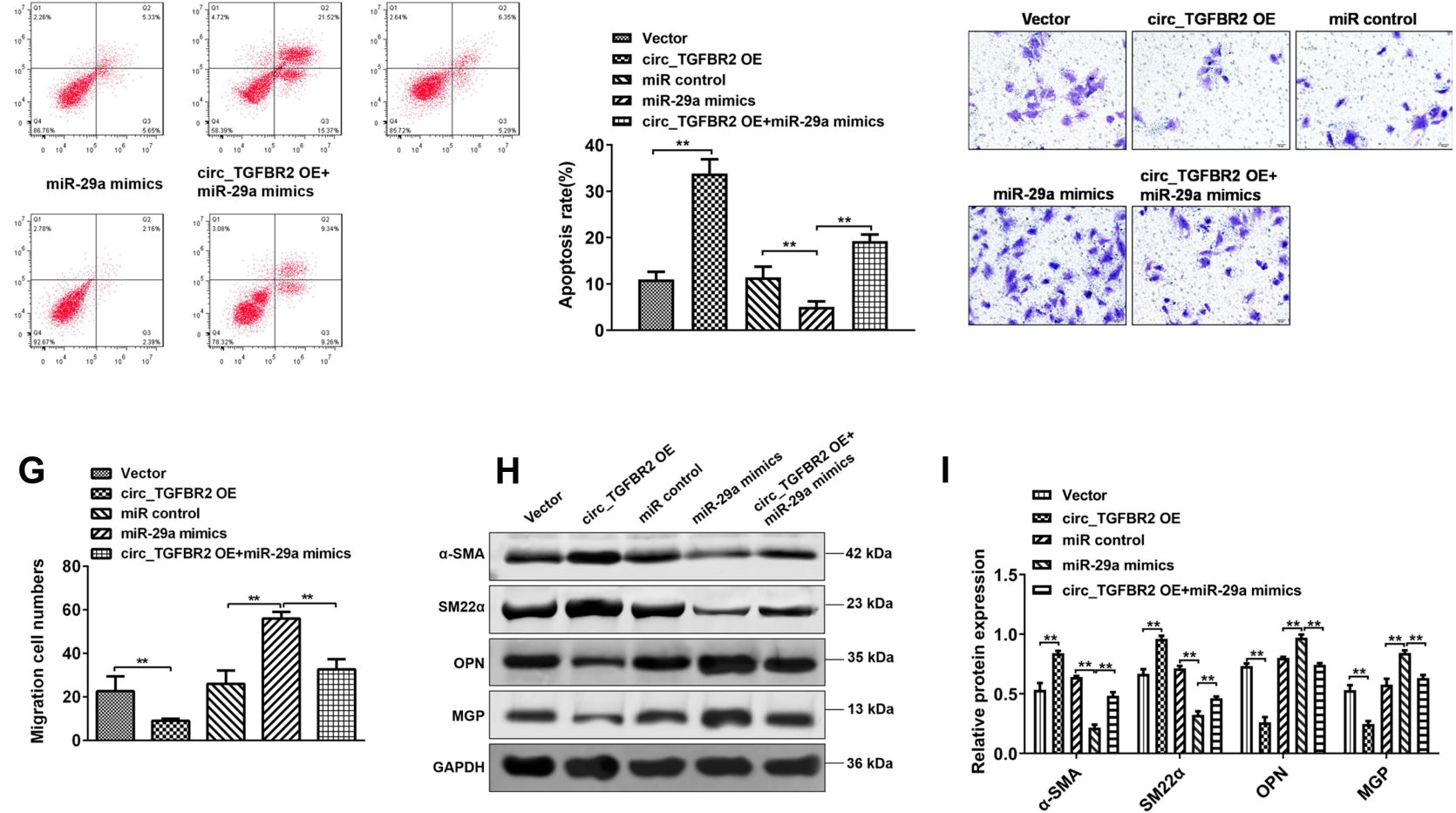

Figure 5 miR-29a mimics significantly rescued circ_TGFBR2-mediated regulation of biological functions. (A) CCK-8 assay was detected the examine the AD-VSMCs cells proliferation under different treatment $(n=3)$. (B and $\mathbf{C})$ Clonality of AD-VSMCs cells was verified by colony-formation assay ( $n=3)$. $(\mathbf{D}$ and $\mathbf{E})$ Flow cytometry was used to measure cell apoptosis of AD-VSMCs cells co-transfected with circ_TGFBR2 overexpression plasmids or miR-29a mimics ( $\mathrm{n}=3$ ). (F and $\mathbf{G})$ Transwell assay was utilized to detect migration of AD-VSMCs cells transfected with indicative plasmids $(n=3)$. ( $\mathbf{H}$ and $\mathbf{I})$ Western blot assay demonstrated the expression of phenotype switch-related proteins expression of AD-VSMCs cells. $*_{p}^{*}<0.0 \mathrm{I}$, ns $=$ non-significant in Tukey's post-hoc comparisons test $(\mathbf{A}, \mathbf{B}, \mathbf{E}, \mathbf{G}$ and $\mathbf{I})$.

SMA, SM22 $\alpha$ ) and synthetic markers (MGP, OPN) (Figure 5H and I).

\section{miR-29a Directly Targeted KLF4 in AD}

To clarify the precise mechanism underlying the biological regulation induced by circ_TGFBR2, we studied the downstream targets of miR-29a through miRDB, miRWalk, miR-tarbase and TargetScan database. According the analysis, KLF4 was selected as a candidate (Figure 6A). Figure 6B showeds the target region of miR-29a and KLF4. Here, to identify the interaction between miR-29a and KLF4, we conducted luciferase reporter assay after co-transfection with wild-type (WT) or mutated (MUT) KLF4-3'UTR and miR-29a mimics or mimics control. The results showed that miR29a only markedly inhibited the luciferase intensity of cotransfection with WT KLF4-3'UTR compared with other groups (Figure 6C). RNA pull-down assay was applied to further determine the interaction between miR-29a and KLF4. As indicated by the findings, miR-29a expression 


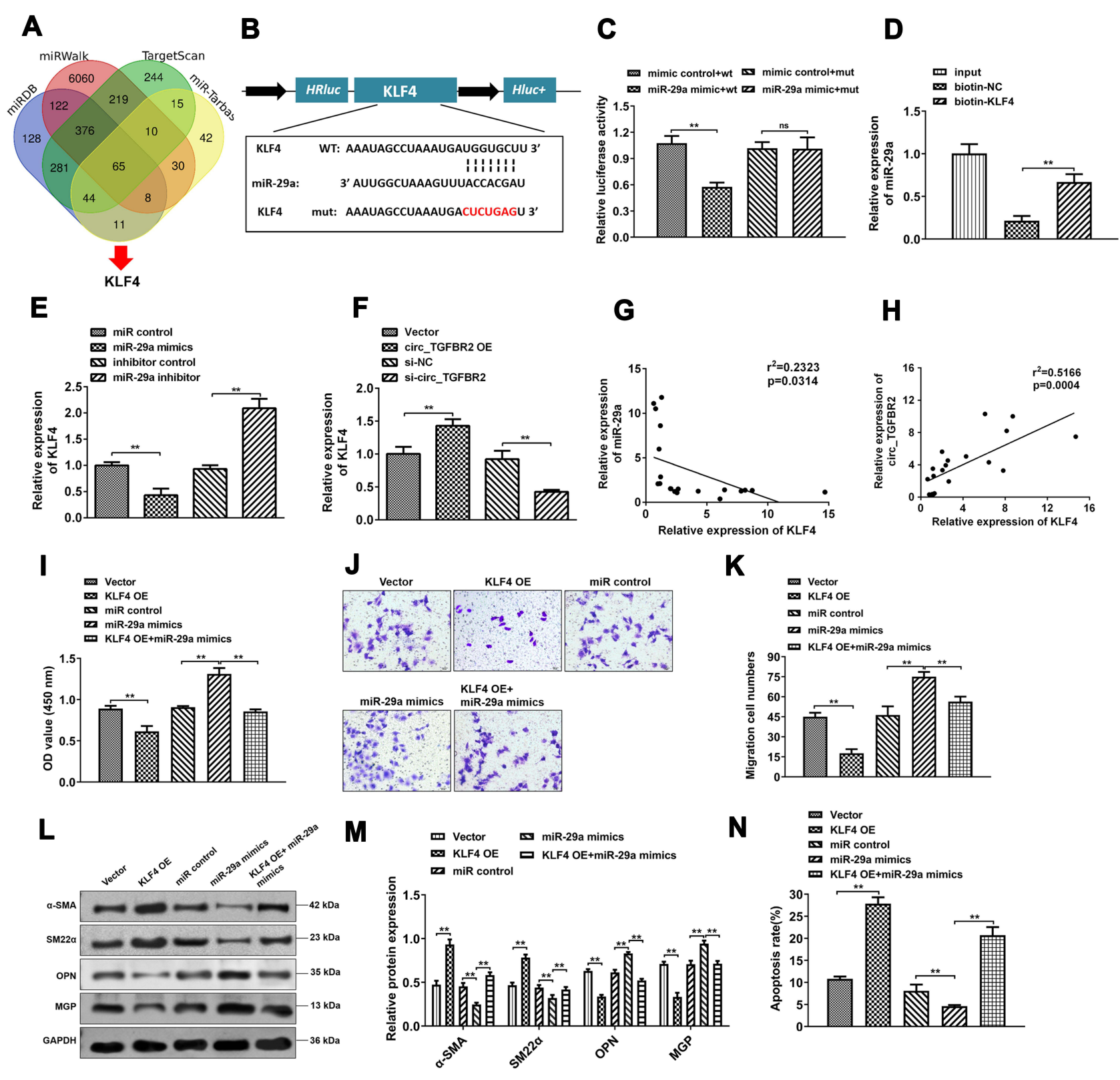

Figure 6 KLF4 acted as a molecular target of miR-29a. (A) The potential target genes of miR-29a were predicted by miRDB, miRWalk, miR-tarbase and TargetScan database. (B) The predicted wild-type (WT) or mutated (MUT) miR-29a binding sites in the 3'-UTR of KLF4 mRNA. (C and D) The binding between miR-29a and KLF4 was determined through Luciferase activity assay $(n=3)(\mathbf{C})$ and RNA pull down $(n=3)(\mathbf{D})$. (E and $\mathbf{F})$ qRT-PCR assay was detected to determine the KLF4 mRNA expression level in AD-VSMCs cells that transfected with miR-29a mimics or inhibitor $(n=3)(\mathbf{E})$, circ TGFBR2 siRNA or overexpression plasmids $(n=3)(\mathbf{F})$. $(\mathbf{G}$ and $\mathbf{H})$ Pearson analysis was applied to examine the correlation between miR-29a and KLF4 $(n=20)(\mathbf{G})$ as well as circ_TGFBR2 and KLF4 ( $n=20)$ in clinical AD samples $(\mathbf{H})$. P < 0.05 for the correlations, by Pearson correlation coefficient analysis. (I) The proliferation of AD-VSMCs cells was measured by CCK-8 assay ( $\mathrm{n}=3$ ). (J and K) The migration ability of AD-VSMCs cells was determined by transwell assay $(n=3)$. ( $\mathbf{L}$ and $\mathbf{M})$ The expression of contractile markers $(\alpha-S M A$, SM22 $\alpha)$ and synthetic markers $(M G P$, OPN) were detected through Western blot assay. (N) The apoptotic cells were detected through flow cytometry.** $p<0.0 \mathrm{I}$, ns $=$ non-significant in 2 -tailed $t$-test $(\mathbf{C})$ and Tukey's posthoc comparisons test (D, E, F, I, K, $\mathbf{M}$ and $\mathbf{N}$ ).

was enriched by biotin-labeled KLF4 in AD-VSMCs cells (Figure 6D). In addition, qRT-PCR analysis was conducted to examine KLF4 expression after miR-29a or circ_TGFBR2 up/down regulation. The results showed that KLF4 level inhibited after miR-29a mimics or circ_TGFBR2 siRNA transfection whereas KLF4 expression increased obviously after miR-29 inhibitor or circ_TGFBR2 overexpression plasmids transfection (Figure 6E and F). Pearson analysis revealed the significant negative correlation between miR-29a and KLF4, and a positive correlation between circ_TGFBR2 and KLF4 (Figure 6G and H). Further studies revealed that KLF4 
overexpression in AD-VSMCs cells effectively inhibited the proliferation (Figure 6I), migration (Figure 6K and L) and the expression of synthetic markers (MGP, OPN) (Figure 6M and $\mathrm{N}$ ), whereas increased the apoptosis rate (Figure $6 \mathrm{~N}$ ) and the expression of contractile markers $(\alpha$ SMA, SM22 $\alpha$ ). However, the biological functions induced by overexpression of KLF4 remarkably overturned by cotransfection of KLF4 overexpression plasmid and miR-29a mimics (Figure 6I-N).

\section{circ_TGFBR2 Regulated the Promotion of $A D$ in vivo}

Next, we carried out in vivo study to further investigate the biological functions of circ_TGFBR2 in the progression of $\mathrm{AD}$. We constructed $\mathrm{AD}$ animal models and as shown in Figure 7A, compared with the Sham group, HE staining results revealed that muscle fiber assembly was disordered and the membrane thickness of the AD aorta increased notably. RT-PCR results showed that the expression of circ_TGFBR2 in AD aorta samples was obviously downregulated compared with the Sham group (Figure 7B). Then, the AD model rats are grouped and treated as indicated in Figure 7C. The IHC staining was applied to determine the Ki67 expression in aorta tissues and the results revealed that injection of $\mathrm{AD}$ rats with lentivirus carrying sicirc_TGFBR2 could notably promote Ki67 expression while overexpression of circ_TGFBR2 remarkably inhibited the Ki67 expression (Figure 7D and E). Besides, as

A $\quad$ B
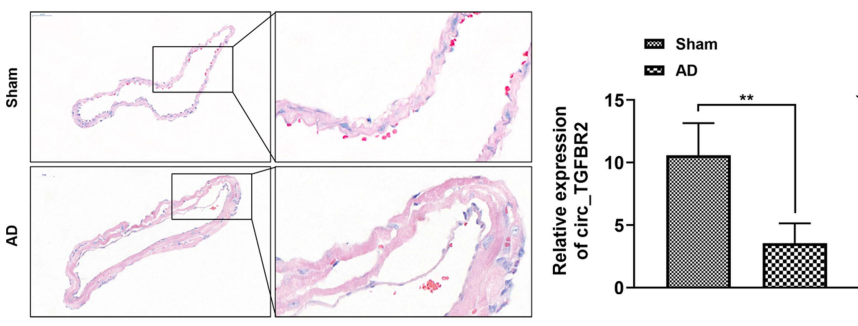

E

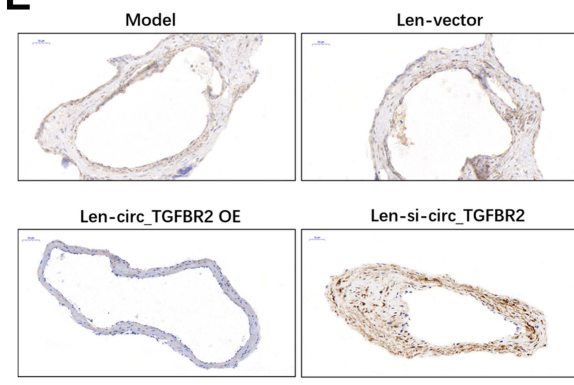

H $m$ Model $m$ Len-circ_tGFBR2 OE Len-Vector m Len-si-circ_TGFBR2
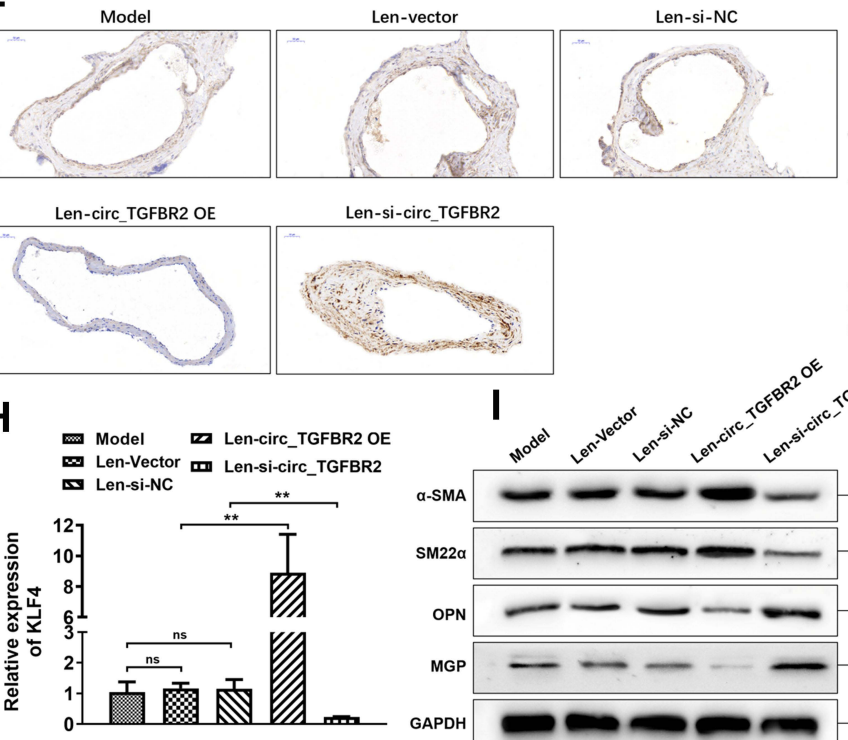

$\mathbf{F}$

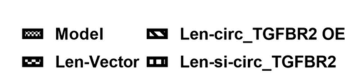

ㅎำ

Len-si-NC

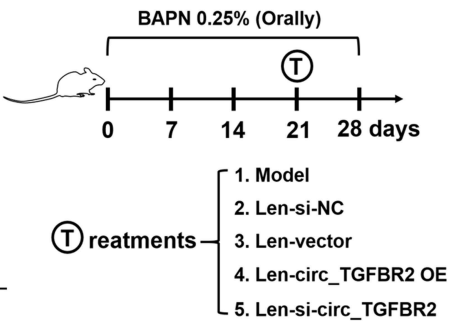

D
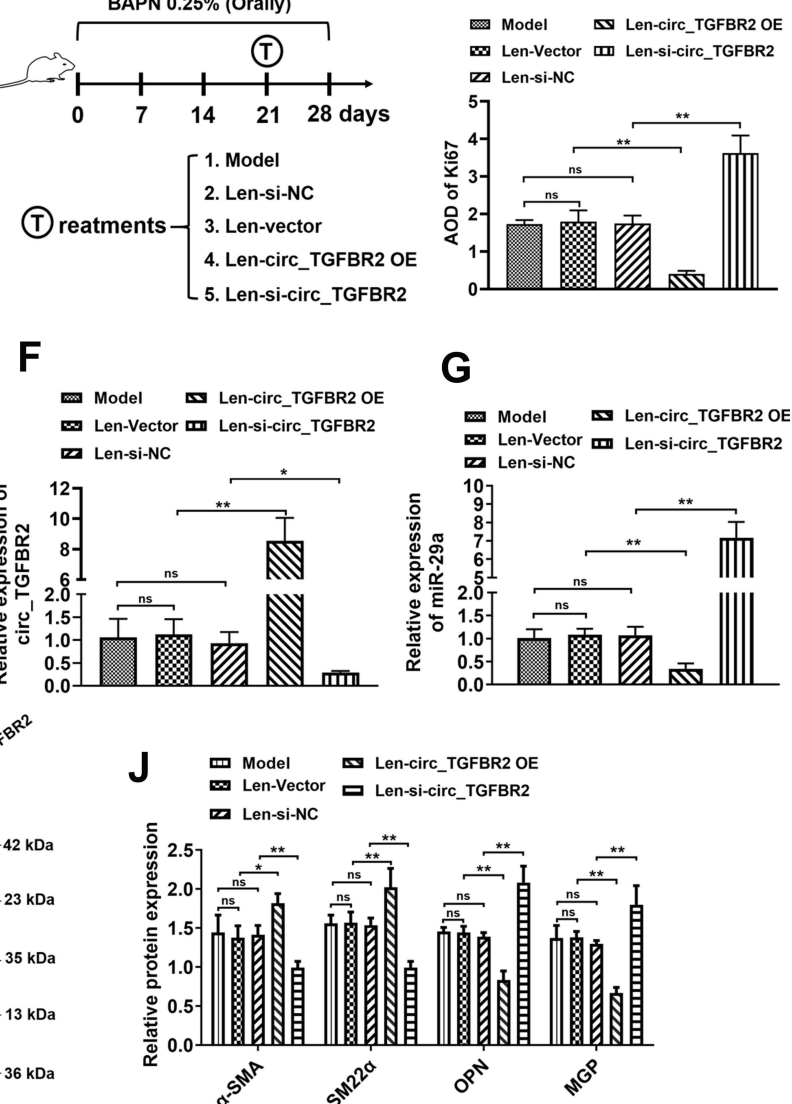

Figure 7 circ_TGFBR2 regulated the promotion of AD in vivo. (A) HE staining results showed the obvious vascular media degeneration and the disordered muscle fibers assembly with the increased middle membrane thickness in the AD group compared with the Sham group $(n=3)$. Scale bar $=50 \mu m$. $(B)$ qRT-PCR analysis revealed that the level of circ_TGFBR2 was down-regulated in AD samples compared with the Sham group $(n=5)$. (C) A schematic diagram showing the process of AD model construction and the time points and treatments of each group. AD rat model was orally administrated by BAPN (0.25\%) for 28 days and at 21 days after BAPN administration, each group was treated as indicated. ( $\mathbf{D}$ and $\mathbf{E})$ Immunohistochemistry analysis was applied to assess the expression of Ki67 in aorta tissues $(n=3)$. Scale bar $=50 \mu m$. $(\mathbf{F}-\mathbf{H})$ qRT-PCR analysis was carried out to evaluate the expression level of circ_TGFBR2 (F), miR-29a (G), and KLF4 (H) of aorta tissues ( $n=5)(\mathbf{I}$ and $\mathbf{J})$ Phenotype switch-related proteins expression of aorta tissues was identified via Western Blot analysis $(n=3)$. ${ }^{*} p<0.05$, ${ }^{* *} p<0.01$, ns $=$ non-significant in 2 -tailed $t$-test $(\mathbf{B})$ and Tukey's post-hoc comparisons test (D, F, G, $\mathbf{H}$ and $\mathbf{J})$.

Abbreviations: BAPN, $\beta$-Aminopropionitrile monofumarate; Len-si-NC, lentivirus carrying si-NC; Len-si-circ_TGFBR2, lentivirus carrying small interfering RNA targeting circ_TGFBR2; Len-vector, lentivirus carrying blank vector; Len-circ_TGFBR2 OE, lentivirus carrying circ_TGFBR2 overexpression plasmids. 
Figure 7F-H showed, the expression level of circ_TGFBR2 and KLF4 in aorta samples were obviously increased, while the expression of miR-29a was inhibited after circ_TGFBR2 overexpression lentivirus administration. However, inhibition of circ_TGFBR2 accelerated the expression of miR-29a while reduced the expression of circ_TGFBR2 and KLF4. Furthermore, Western blot assay revealed that the expression level of $\alpha$-SMA and SM22 $\alpha$ was enhanced, while the expression level of OPN and MGP was inhibited in aorta tissues after circ_TGFBR2 overexpression. However, the opposite results of the expression of $\alpha$-SMA, SM22 $\alpha$, OPN and MGP were obtained after si-circ _TGFBR2 treatment (Figure 7I and J). Thus, we concluded that circ_TGFBR2 is down-regulated in $\mathrm{AD}$, and its overexpression inhibited the promotion of $\mathrm{AD}$ in vitro and in vivo.

\section{Discussion}

CircRNAs are pivotal regulator in gene expression on the post-transcriptional level. Increasing evidences indicate that circRNAs are dysregulated in multiple cardiovascular diseases. But the biological function and underlying mechanisms of circRNAs in AD are still largely unknown. The main histological features of $\mathrm{AD}$ are phenotypic switch of vascular muscle cells (VSMCs), which transformed from a contractile to a synthetic phenotype. ${ }^{22,23}$ And this switch leads to the enhancement of cells migration and proliferation and reduction of contractility. ${ }^{24}$ Circ_0004872 acted key roles in atherosclerosis (AS) progression. Circ_0004872 promoted PDGF-BB-induced proliferation, cell migration and dedifferentiation in HA-VSMCs cells by increasing TXNIP expression through sponging miR-513a-5P. ${ }^{25}$ Circ_Lrp6 suppressed miR-145-mediated the migration and differentiation of VSMCs cells and inhibition of circ_Lrp6 expression prevented the occurrence of intimal hyperplasia. ${ }^{26}$ In this research, we determined a novel circRNA circ_TGFBR2. We determined that circ_TGFBR2 originates from exon 4 of TGFBR2 and generates a loop structure through joining the $3^{\prime}$ tail and 5' cap. Stability of circ_TGFBR2 was confirmed by its stable expression under RNase $\mathrm{R}$ digestion. As far as we know, this is the first study of circ_TGFBR2 focused on its altered expression and biological effect in $\mathrm{AD}$.

CircRNAs are a special group of novel endogenous IncRNAs that can bind to and interact with miRNAs and play an important regulatory role at the transcriptional or post-transcriptional level. ${ }^{27,28}$ Otherwise, circRNAs like circRNA-101238, ${ }^{29}$ circ-MARK ${ }^{30}$ have been reported and studied in AD. The up-regulation of miR-233 has been found in aorta tissues of ApoE knockout mice and involved with the increased VSMCs cells migration and calcification. ${ }^{31}$ MiR137 exerted a negative regulation in VSMCs cells dedifferentiation and proliferation in simulated microgravity rats through mediating calcineurin/NFAT pathway. ${ }^{32}$ Herein, we demonstrated that circ_TGFBR2 mainly located in cytoplasm through FISH assay, thus indicating that circ_TGFBR2 may function as miRNA sponges. The bioinformatics analysis showed that circ_TGFBR2 has potential binding sites for plenty of miRNAs. And the RNA pull-down and luciferase reporter assays determined that binding interaction between circ_TGFBR2 and miR-29a. Moreover, function studies revealed miR-29a overexpression reversed the effect of circ_TGFBR2 which further verified the relation between them. Circ_TGFBR2 exerted AD disease suppressor functions through ceRNA mechanism to miR-29a and rescued the inhibition of miR-29a on its target gene KLF4.

KLF4, or Kruppel-like factor 4 , is a member zinc finger-containing transcription factor that regulates multiple cellular processes like cell proliferation, cell migration and differentiation. ${ }^{33}$ Previous research had revealed that KLF4 gene was relevant with various vascular diseases, including abdominal aortic aneurysms and atherosclerosis. $^{34,35}$ Also, KLF4 has been identified as a key factor required for VSMCs cells phenotypic modulation. ${ }^{36}$ For the function of KLF4 in VSMCs phenotype switch, there are two conflicting opinions: KLF4 inhibits VSMCs phenotype switch and KLF4 promotes VSMCs phenotype regulation. Zeng et $\mathrm{al}^{37}$ found that the PDGF-induced VSMCs phenotypic modulation was accompanied by the increase of KLF4. And after inhibition of KLF4, the VSMCs differentiation induced by PDGF was abrogated. NOXA1-dependent NAPDH oxidase enhanced VSMCs invasion and reduced KLF4mediated transition to macrophage-like cells and plaque inflammation during restenosis and atherosclerosis in Noxa1-/- mice. ${ }^{38}$ Gsa deletion decreased cAMP level and increased HuR expression, which binds to the adenylate uridylate-rich elements of the $3^{\prime}$ untranslated region of KLF4 mRNA, thus regulating angiotensin II-induced abdominal aortic aneurysm formation. ${ }^{34}$ In our study, our results suggested that circ_TGFBR2/miR-29a/KLF4 axis mediated AD-VSMCs proliferation, migration and phenotypic switching. Although the upregulation of KLF4 by circ_TGFBR2 mediates AD promotion, more mechanistic details about KLF4 involved are still needed and will be our next research stage. 
In conclusion, our findings revealed that circ_TGFBR2 was down-regulated in $\mathrm{AD}$ on vivo and vitro level. Mechanistically, circ_TGFBR2 sponged miR-29a in ADVSMC cells to regulate proliferation, migration and VSMCs phenotypic switch by affecting expression of target KLF4. However, there are still some limits in our study. The expression level of circ_TGFBR2 needs to be validated in more clinical samples. Also, what regulates circ_TGFBR2 to work should be illustrated in the future.

\section{Acknowledgments}

The research was supported by the Youth Program of National Natural Science Foundation of China (Grant No. 81800408) and the Key medical science and technology development project in Nanjing (Grant No. ZKX17021) and The Six talent peaks project in Jiangsu Province (Grant No. WSW-142).

\section{Author Contributions}

All authors made a significant contribution to the work reported, whether that is in the conception, study design, execution, acquisition of data, analysis and interpretation, or in all these areas; took part in drafting, revising or critically reviewing the article; gave final approval of the version to be published; have agreed on the journal to which the article has been submitted; and agree to be accountable for all aspects of the work.

\section{Disclosure}

There is no conflict of interest to declare in this research.

\section{References}

1. Clough RE, Nienaber CA. Management of acute aortic syndrome. Nat Rev Cardiol. 2015;12:103-114. doi:10.1038/nrcardio.2014.203

2. Nienaber CA, Clough RE, Sakalihasan N, et al. Aortic dissection. Nat Rev Dis Primers. 2016;2:16053. doi:10.1038/nrdp.2016.53

3. Silaschi M, Byrne J, Wendler O. Aortic dissection: medical, interventional and surgical management. Heart. 2017;103:78-87. doi:10.1136/ heartjnl-2015-308284

4. Ignatieva E, Kostina D, Irtyuga $\mathrm{O}$, et al. Mechanisms of smooth muscle cell differentiation are distinctly altered in thoracic aortic aneurysms associated with bicuspid or tricuspid aortic valves. Front Physiol. 2017;8:536. doi:10.3389/fphys.2017.00536

5. Wang Y, Dong CQ, Peng GY, et al. MicroRNA-134-5p regulates media degeneration through inhibiting VSMC phenotypic switch and migration in thoracic aortic dissection. Mol Ther Nucleic Acids. 2019;16:284-294. doi:10.1016/j.omtn.2019.02.021

6. Frismantiene A, Philippova M, Erne P, Resink TJ. Smooth muscle cell-driven vascular diseases and molecular mechanisms of VSMC plasticity. Cell Signal. 2018;52:48-64. doi:10.1016/j.cellsig.2018.08.019
7. Rangrez AY, Massy ZA, Metzinger-le Meuth V, Metzinger L. miR143 and miR-145: molecular keys to switch the phenotype of vascular smooth muscle cells. Circ Cardiovasc Genet. 2011;4:197-205. doi:10.1161/CIRCGENETICS.110.958702

8. Davis-Dusenbery BN, Wu C, Hata A. Micromanaging vascular smooth muscle cell differentiation and phenotypic modulation. Arterioscler Thromb Vasc Biol. 2011;31:2370-2377. doi:10.1161/ ATVBAHA.111.226670

9. Gong L, Zhou X, Sun J. Circular RNAs interaction with MiRNAs: emerging roles in breast cancer. Int J Med Sci. 2021;18:3182-3196. doi:10.7150/ijms.62219

10. Lu TX, Rothenberg ME. MicroRNA. J Allergy Clin Immunol. 2018;141:1202-1207. doi:10.1016/j.jaci.2017.08.034

11. Du WW, Zhang C, Yang W, Yong T, Awan FM, Yang BB. Identifying and characterizing circRNA-protein interaction. Theranostics. 2017;7:4183-4191. doi:10.7150/thno.21299

12. Sun Y, Yang Z, Zheng B, et al. A novel regulatory mechanism of smooth muscle $\alpha$-actin expression by NRG-1/circACTA2/miR-548f5p axis. Circ Res. 2017;121:628-635. doi:10.1161/CIRCRES AHA.117.311441

13. Yue J, Zhu T, Yang J, et al. CircCBFB-mediated miR-28-5p facilitates abdominal aortic aneurysm via LYPD3 and GRIA4. Life Sci. 2020;253:117533. doi:10.1016/j.lfs.2020.117533

14. Takeda N, Hara H, Fujiwara T, Kanaya T, Maemura S, Komuro I. TGF- $\beta$ signaling-related genes and thoracic aortic aneurysms and dissections. Int $J$ Mol Sci. 2018;19(7):2125. doi:10.3390/ ijms 19072125

15. Hu JH, Wei H, Jaffe M, et al. Postnatal deletion of the type II transforming growth factor- $\beta$ receptor in smooth muscle cells causes severe aortopathy in mice. Arterioscler Thromb Vasc Biol. 2015;35 (12):2647-2656. doi:10.1161/ATVBAHA.115.306573

16. Hadrava V, Tremblay J, Hamet P. Abnormalities in growth characteristics of aortic smooth muscle cells in spontaneously hypertensive rats. Hypertension. 1989;13:589-597. doi:10.1161/01.HYP.13.6.589

17. Bruel A, Ortoft G, Oxlund H. Inhibition of cross-links in collagen is associated with reduced stiffness of the aorta in young rats. Atherosclerosis. 1998;140:135-145. doi:10.1016/S0021-9150(98) 00130-0

18. Nakashima Y, Sueishi K. Alteration of elastic architecture in the lathyritic rat aorta implies the pathogenesis of aortic dissecting aneurysm. Am J Pathol. 1992;140:959-969.

19. Xiao Y, Sun Y, Ma X, et al. MicroRNA-22 inhibits the apoptosis of vascular smooth muscle cell by targeting p38MAPK $\alpha$ in vascular remodeling of aortic dissection. Mol Ther Nucleic Acids. 2020;22:1051-1062. doi:10.1016/j.omtn.2020.08.018

20. Wu Z, Chang J, Ren W, Hu Z, Li B, Liu H. Bindarit reduces the incidence of acute aortic dissection complicated lung injury via modulating NF-кB pathway. Exp Ther Med. 2017;14:2613-2618. doi:10.3892/etm.2017.4830

21. Kristensen LS, Andersen MS, Stagsted LVW, Ebbesen KK, Hansen TB, Kjems J. The biogenesis, biology and characterization of circular RNAs. Nat Rev Genet. 2019;20:675-691. doi:10.1038/ s41576-019-0158-7

22. Michel JB, Jondeau G, Milewicz DM. From genetics to response to injury: vascular smooth muscle cells in aneurysms and dissections of the ascending aorta. Cardiovasc Res. 2018;114:578-589. doi:10.1093/cvr/cvy006

23. Rzucidlo EM, Martin KA, Powell RJ. Regulation of vascular smooth muscle cell differentiation. J Vasc Surg. 2007;45(Suppl A):A25-A32. doi:10.1016/j.jvs.2007.03.001

24. Mao N, Gu T, Shi E, Zhang G, Yu L, Wang C. Phenotypic switching of vascular smooth muscle cells in animal model of rat thoracic aortic aneurysm. Interact Cardiovasc Thorac Surg. 2015;21:62-70. doi:10.1093/icvts/ivv074 
25. Fan K, Ruan X, Wang L, Lu W, Shi Q, Xu Y. Circ 0004872 promotes platelet-derived growth factor-BB-induced proliferation, migration and dedifferentiation in HA-VSMCs via miR-513a-5p/ TXNIP axis. Vascul Pharmacol. 2021;140:106842. doi:10.1016/j. vph.2021.106842

26. Hall IF, Climent M, Quintavalle M, et al. Circ_Lrp6, a circular RNA enriched in vascular smooth muscle cells, acts as a sponge regulating miRNA-145 function. Circ Res. 2019;124(4):498-510. doi:10.1161/ CIRCRESAHA.118.314240

27. Dori M, Bicciato S. Integration of bioinformatic predictions and experimental data to identify circRNA-miRNA associations. Genes. 2019;10:642. doi:10.3390/genes10090642

28. Sun Z, Chen C, Su Y, et al. Regulatory mechanisms and clinical perspectives of circRNA in digestive system neoplasms. J Cancer. 2019;10:2885-2891. doi:10.7150/jca.31167

29. Zou M, Huang C, Li X, et al. Circular RNA expression profile and potential function of hsa_circRNA_101238 in human thoracic aortic dissection. Oncotarget. 2017;8:81825-81837. doi:10.18632/ oncotarget.18998

30. Tian C, Tang X, Zhu X, et al. Expression profiles of circRNAs and the potential diagnostic value of serum circMARK3 in human acute Stanford type A aortic dissection. PLoS One. 2019;14:e0219013. doi:10.1371/journal.pone.0219013

31. Rangrez AY, M'Baya-Moutoula E, Metzinger-le Meuth V, et al. Inorganic phosphate accelerates the migration of vascular smooth muscle cells: evidence for the involvement of miR-223. PLoS One. 2012;7(10):e47807. doi:10.1371/journal.pone.0047807
32. Zhang B, Chen L, Bai YG, et al. miR-137 and its target T-type Ca V 3.1 channel modulate dedifferentiation and proliferation of cerebrovascular smooth muscle cells in simulated microgravity rats by regulating calcineurin/NFAT pathway. Cell Prolif. 2020;53(3): e12774. doi:10.1111/cpr.12774

33. Ghaleb AM, Yang VW. Krüppel-like factor 4 (KLF4): what we currently know. Gene. 2017;611:27-37. doi:10.1016/j.gene.2017.02.025

34. Qin X, He L, Tian M, et al. Smooth muscle-specific Gs $\alpha$ deletion exaggerates angiotensin II-induced abdominal aortic aneurysm formation in mice in vivo. $J$ Mol Cell Cardiol. 2019;132:49-59. doi:10.1016/j.yjmcc.2019.05.002

35. Yoshida T, Kaestner KH, Owens GK. Conditional deletion of Krüppel-like factor 4 delays downregulation of smooth muscle cell differentiation markers but accelerates neointimal formation following vascular injury. Circ Res. 2008;102:1548-1557. doi:10.1161/ CIRCRESAHA.108.176974

36. Zheng B, Han M, Wen JK. Role of Krüppel-like factor 4 in phenotypic switching and proliferation of vascular smooth muscle cells. IUBMB Life. 2010;62:132-139.

37. Zeng Q, Wei B, Zhao Y, et al. Shh mediates PDGF-induced contractile-to-synthetic phenotypic modulation in vascular smooth muscle cells through regulation of KLF4. Exp Cell Res. 2016;345:82-92. doi:10.1016/j.yexcr.2016.05.014

38. Vendrov AE, Sumida A, Canugovi C, et al. NOXA1-dependent NADPH oxidase regulates redox signaling and phenotype of vascular smooth muscle cell during atherogenesis. Redox Biol. 2019;21:101063. doi:10.1016/j.redox.2018.11.021
Journal of Inflammation Research

\section{Publish your work in this journal}

The Journal of Inflammation Research is an international, peerreviewed open-access journal that welcomes laboratory and clinical findings on the molecular basis, cell biology and pharmacology of inflammation including original research, reviews, symposium reports, hypothesis formation and commentaries on: acute/chronic inflammation; mediators of inflammation; cellular processes; molecular mechanisms; pharmacology and novel anti-inflammatory drugs; clinical conditions involving inflammation. The manuscript management system is completely online and includes a very quick and fair peerreview system. Visit http://www.dovepress.com/testimonials.php to read real quotes from published authors. 